\title{
Efficacy of Mindfulness-based Stress Reduction in Hemodialysis Patients with Anxiety and Depression: a randomized, double-blind, parallel-group trial
}

Mostafa Haghshenas ${ }^{1}$, Fatemeh Assarian², Abdollah Omidi ${ }^{3}$, Mohammadreza Razaghof ${ }^{4}$, Habibollah Rahimi ${ }^{5}$

${ }^{1}$ M.D., Department of Psychiatry, Faculty of Medicine, Kashan University of Medical Sciences, Kashan, Iran

${ }^{2}$ M.D., Associate Professor, Department of Psychiatry, Faculty of Medicine, Kashan University of Medical Sciences, Kashan, Iran

${ }^{3}$ Ph.D. of Clinical Psychology, Associate Professor, Faculty of Medicine, Kashan University of Medical Sciences, Kashan, Iran

${ }^{4}$ M.D., Faculty of Medicine, Tehran University of Medical Sciences, Tehran, Iran

${ }^{5} \mathrm{Ph} . \mathrm{D}$. of Epidemiology, Clinical Research Development Unit - Matini/Kargarnejad Hospitals, Kashan University of Medical Sciences, Kashan, Iran

\section{Type of article: Original}

\begin{abstract}
Background: Diseases with a chronic and debilitating nature, such as chronic kidney disease, are frequently associated with psychiatric disorders like anxiety and depression.

Objective: To assess the efficacy of mindfulness-based stress reduction (MBSR) on anxiety and depression in hemodialysis patients.

Methods: In this randomized clinical trial, fifty hemodialysis patients were selected from two dialysis centers of Kashan city in Iran in 2016. The patients had desired cognitive status based on MMSE (Mini Mental Status Examination) and clinical interview, and were randomly allocated into the intervention and control groups. The intervention group $(\mathrm{n}=25)$ received a Mindfulness-Based Stress Reduction (MBSR) program in addition to the health education program in eight sessions, whereas the control group $(n=25)$ received only the health education program. To measure the anxiety and depression score before and after study, the Hospital Anxiety and Depression Scale were used. Data were analyzed by SPSS version 11.5, using Analysis of Covariance (ANCOVA), t-test, Chi-square, Mann-Whitney U, and Fisher exact test.

Results: The mean age of intervention and control groups were $46.86 \pm 11.66$ and $46.26 \pm 11.71$ respectively. The mean duration of illness was $2.47 \pm 1.78$ and $2.62 \pm 1.32$ in intervention and control groups respectively. There were no significant differences between the two groups regarding age, sex, education level and job. Depression and anxiety were reduced in the intervention group compared with the control group $(\mathrm{p}=0.0001)$.

Conclusions: The MBSR program may reduce anxiety and depression in hemodialysis patients.

Funding: This study was financially supported by the Deputy of Research, Kashan University of Medical Sciences, Kashan, Iran (Ref: 9436).

Clinical Trial Registration: The protocol of study was registered at the Iranian Registry of Clinical Trials (IRCT) with ID number of IRCT2015081723652N1.

Keywords: Mindfulness, Anxiety, Depression, Renal dialysis
\end{abstract}

\section{Corresponding author:}

Associate Professor Dr. Fatemeh Assarian, Department of Psychiatry, Faculty of Medicine, Kashan University of Medical Sciences, Kashan, I.R. Iran. Tel: +98.3155549111, Fax: +98.3155540036, Email: fa_assar@yahoo.com

Received: June 12, 2018, Accepted: September 28, 2018, Published: March 2019

iThenticate screening: February 13, 2019, English editing: February 19, 2019, Quality control: February 21, 2019

This article has been reviewed / commented by five experts

Funding / research project approval: Kashan University of Medical Sciences (Ref: 9436)

Ethics approval: Kashan University of Medical Sciences (Ref: IR. KAUMS 1394.36)

(C) 2019 The Authors. This is an open access article under the terms of the Creative Commons Attribution-NonCommercialNoDerivs License, which permits use and distribution in any medium, provided the original work is properly cited, the use is non-commercial and no modifications or adaptations are made. 
Note: This study has followed the CONSORT Statement, which is an evidence-based, minimum set of recommendations for reporting randomized trials. It offers a standard way for authors to prepare reports of trial findings, facilitating their complete and transparent reporting, and aiding their critical appraisal and interpretation.

\section{Introduction}

Chronic kidney disease (CKD) is considered one of the major health issues worldwide. Over the past two decades, the prevalence of CKD has been increasing in both developed and developing countries (1-3). More than 60 thousand people die of kidney diseases around the world annually (4). The incidence of this disease in different regions of the world varies; but in general, the incidence of this disease in most countries is more than 200 per million (5). Hemodialysis is the most common therapy and intended to maximally normalize the patient's life. The number of hemodialysis patients increases $15 \%$ in Iran every year (6). Hemodialysis is a stressful process and is associated with multiple social and psychological problems predisposing to mental disorders. Studies have shown that the most common psychological problems in hemodialysis patients are depression and anxiety (7). In a study by Nazemian, the prevalence of depression, anxiety and hidden stress was reported as $64.5 \%, 51.4 \%$, and $49.7 \%$ respectively (8). According to the Nazemian study, depression can lead to hospitalization in these patients, worsen the prognosis, and increase the mortality rate (8). Depression can also be associated with suicidal ideation and poor dialysis compliance in these patients (9).

Anxiety, as the second common psychological problem in hemodialysis patients, has been studied less frequently. However, anxiety can affect the quality of life and treatment compliance of patients (10). This has further turned the attention of clinicians toward the diagnosis of psychiatric problems of hemodialysis patients and the use of medical and psychological therapies for them (11). Nevertheless, as the efficacy and safety of Selective Serotonin Reuptake Inhibitors (SSRIs, such as citalopram) for depression in CKD patients have yet to be accepted by clinicians, its implication is relatively low and accompanied with adverse effects, such as high protein-binding, aggravated nausea, and increased bleeding tendency, which can be dangerous in CKD patients (11). Tricyclic antidepressants (such as Amitriptyline and Nortiliptyline) and Monoamine oxidase inhibitors (such as Tranylcypromine) should be used with caution due to their potential side effects (e.g., arrhythmias, drug-drug interaction, hypotension) that are exacerbated in dialysis patients. SNRIs such as venlafaxine are excreted via kidneys and are not significantly removed by dialysis and may cause increased hypertension (12).

Over the past two decades, one of the psychological interventions to improve the mental health of patients with chronic diseases, which has come to attention, is the mindfulness-based stress reduction (MBSR) program. Mindfulness is a new concept in the field of psychotherapy, which has been greatly welcomed by clinicians during the past decade (13). Mindfulness aids the patient to change their behavior towards environmental events by informing the patient of the events that are being experienced by his mind and body at present. From this point of view, one cannot control all the stressful events around the patient, but one can act more efficiently by changing the response the patient shows to those events (14). Increasing one's awareness of what one experiences at every moment and creating the spirit of acceptance and compassion, mindfulness helps people not to only change the relationship between themselves and their internal experiences but also to confront the stressful events more efficiently (15). To our knowledge, the efficacy of this intervention has not been studied in hemodialysis patients so far. Nonetheless, it has been studied in other chronic diseases including chronic pain, multiple sclerosis, cancer and cardiopulmonary disorders, which had been significant in the improvement of anxiety and depression of these patients (16-18). This study was done to examine the efficacy of MBSR program on anxiety and depression in hemodialysis patients.

\section{Material and Methods}

\subsection{Trial design and participants}

This was a randomized, double-blind, parallel-group trial to determine effect of MBSR on anxiety and depression in hemodialysis patients. It was conducted in Kashan, Iran from March 2015 to September 2015. The participants were chosen through convenience sampling method from Shahid Beheshti hospital and Akhavan hospital, which are the only hemodialysis centers in Kashan city. Sample size calculation was according to results of a previous randomized controlled trial (19). The calculated sample size was 16 patients for each group of intervention and controls. Considering attrition rates, we increased the required sample size to 25 . 


\subsection{Selection criteria}

\subsubsection{Inclusion criteria}

The inclusion criteria were hemodialysis patients referred twice weekly for three to four hours in each session for at least one year, aged 18-60 years, the Mini Mental Status Examination questionnaire (MMSE) score of 25 or more, capable of writing and reading, and their depression or anxiety disorders not due to divorce, grief or familial problems.

\subsubsection{Exclusion criteria}

The exclusion criteria were history of previous suicide attempt or having suicidal thoughts, taking anti-psychiatric drugs or treatment in the past six months and lack of follow up in study of more than two MBSR sessions due to physical disease problems.

\subsection{Data collection, blinding and randomization}

The patient recruitments were conducted by nurses of hospitals to which the patients had been referred.. The study objectives were explained to the patients, and those patients who agreed with participating in the study, were asked to attend their hemodialysis center. At the start of study, a psychiatrist explained the purposes and process of the study to the patients, in more detail. Then, a clinical interview was conducted with them aimed at identifying the candidate patients. The baseline anxiety and depression status of all eligible patients was measured with standard questionnaires. A unique code was assigned for each patient then with permuted block randomization, two groups were made that comprised of 25 patients in each. The psychiatric manager was blinded in random allocation process.

\subsection{Intervention}

The intervention group received the mindfulness-based stress reduction (MBSR) program performed by collaboration of an expert psychiatrist and psychologist in eight 120-minute sessions. The sessions were performed for patients, collectively. It comprised of the following stages:

- Session 1: introduction, mindfulness as a way of living, eating exercises, giving feedback and discussion about eating exercises, body scan, giving feedback and discussion about body scan

- Session 2: yoga, discussion about interaction between mindful activities, home exercises and body scan, discussion about mindful substructures

- Session 3: introduction of sitting meditation, 10-minute guided meditation, giving feedback and discussion about sitting meditation

- Session 4: yoga, 15-minute sitting meditation, five-minute watching and listening exercises

- Session 5: guided walking meditation, STOP (one-minute breathing space)

- Session 6: yoga; sitting meditation: awareness of breathing, body, sounds, and thoughts; introducing troublesome thoughts and memories; three-minute breathing space exercises

- Session 7: yoga; sitting meditation: awareness of breathing, body, sounds, and thoughts; mindfulness and compassion; loving-kindness meditation

- Session 8: conclusion, discussion about how to integrate mindfulness exercises into our lifestyle

At the beginning of intervention, the terms and conditions regarding each session were observed by the patients and the treatment contract was set. The control group received only common medical treatment. The intervention was conducted by an expert, trained, qualified psychologist. The assessment was done before and after the intervention. Following the end of sessions, an MBSR program tutorial was given to patients of the control group.

\subsection{Measurements}

Demographic questionnaire included; age, education level, and job status of the patients, which was filled by selfcompletion. To assess cognitive status of participants, the Mini Mental Status Examination questionnaire (MMSE) was applied. The Hospital Anxiety and Depression questionnaire is a concise sensitive scale to measure the anxiety and depression in a person with physical, mental or even no diseases, which includes seven questions for each anxiety and depression. Each question is scored by a four-score scale of 0 to 3, so that a maximum score of 21 is likely for each subscale of anxiety and depression. A score of 11 or more in each subscale is highly indicative of a serious psychiatric disorder, with 8-10 being intermediate, and 0-7 normal. According to results of a validation study on 167 Iranian women who had breast cancer, the internal consistency of depression and anxiety subscales in terms of Cronbach's alpha have been reported as 0.78 and 0.68 , respectively (20). The questionnaires were filled in two time lags, at the beginning and at the end of study with self-completion method. 


\subsection{Ethics}

The study proposal was approved by the ethic committee of Kashan University of Medical Sciences (Ref: IR. KAUMS 1394.36). The protocol of study was registered at the Iranian Registry of Clinical Trials (IRCT) with ID number of IRCT2015081723652N1. Participating in the study or leaving during the study was voluntary. Informed consent was obtained from all patients. The information sheet included the study objective, its stages, and mentioned that the participants had the right to withdraw from the study at any time.

\subsection{Statistical methods}

Before analysis, the normality distribution of data was checked with a histogram graph, for continuous variables. The comparability of two study groups was evaluated in regard to demographic characteristics. The between-group comparisons were conducted with t-test, Chi square and Fisher exact test, and Mann-Whitney U tests in cases of non-normality. The main analysis was conducted with ANCOVA. The P-value was set on 0.05 . SPSS version 11.5 (SPSS Inc., Chicago, Illinois, USA) was used to analyze the data. To find pure MBSR effect on depression and anxiety level, the baseline scores of these variables applied as the covariate variable in the ANCOVA model. Before conducting ANCOVA, the pre-required assumptions (normality and homogeneity of variance) were checked.

\section{Results}

In this study, 50 hemodialysis patients were allocated into two intervention and control groups ( $\mathrm{n}=25)$. Six participants in the intervention group were excluded due to irregular attendance and medical problems, and also, four participants due to medical problems and lack of follow up (Figure 1). The mean age of completed followed participants was $46.8 \pm 11.6$ years and $46.2 \pm 11.7$ years respectively. Among these, $45 \%$ and $55 \%$ were male, in intervention and control groups. History of disease was $2.47 \pm 1.8$ and $2.62 \pm 1.3$ years for intervention and control groups respectively. There was no significant difference between the two groups in terms of age, sex, education, marital status, job, anxiety and depression score and disease duration. Table 1 shows the demographic characteristics of patients.

According to ANCOVA analysis, the mean \pm SD of depression in MBSR and control groups were $5.9 \pm 2.5$ and $7.4 \pm 5.3(\mathrm{~F}=18, \mathrm{p}<0.001)$. The average score of anxiety was $4.7 \pm 2.8$ and $5.5 \pm 3.2(\mathrm{~F}=24.4, \mathrm{p}<0.001)$. The results of ANCOVA analysis are presented in Tables 2 and 3. As Table 2 shows, the effect size of MBSR on depression presented by Partial Eta Squared- was 0.34. According to Cohen's d cut-off, it is considered as the moderate effect size. This was 0.41 for anxiety, as the moderate effect size too (Table 3 ).

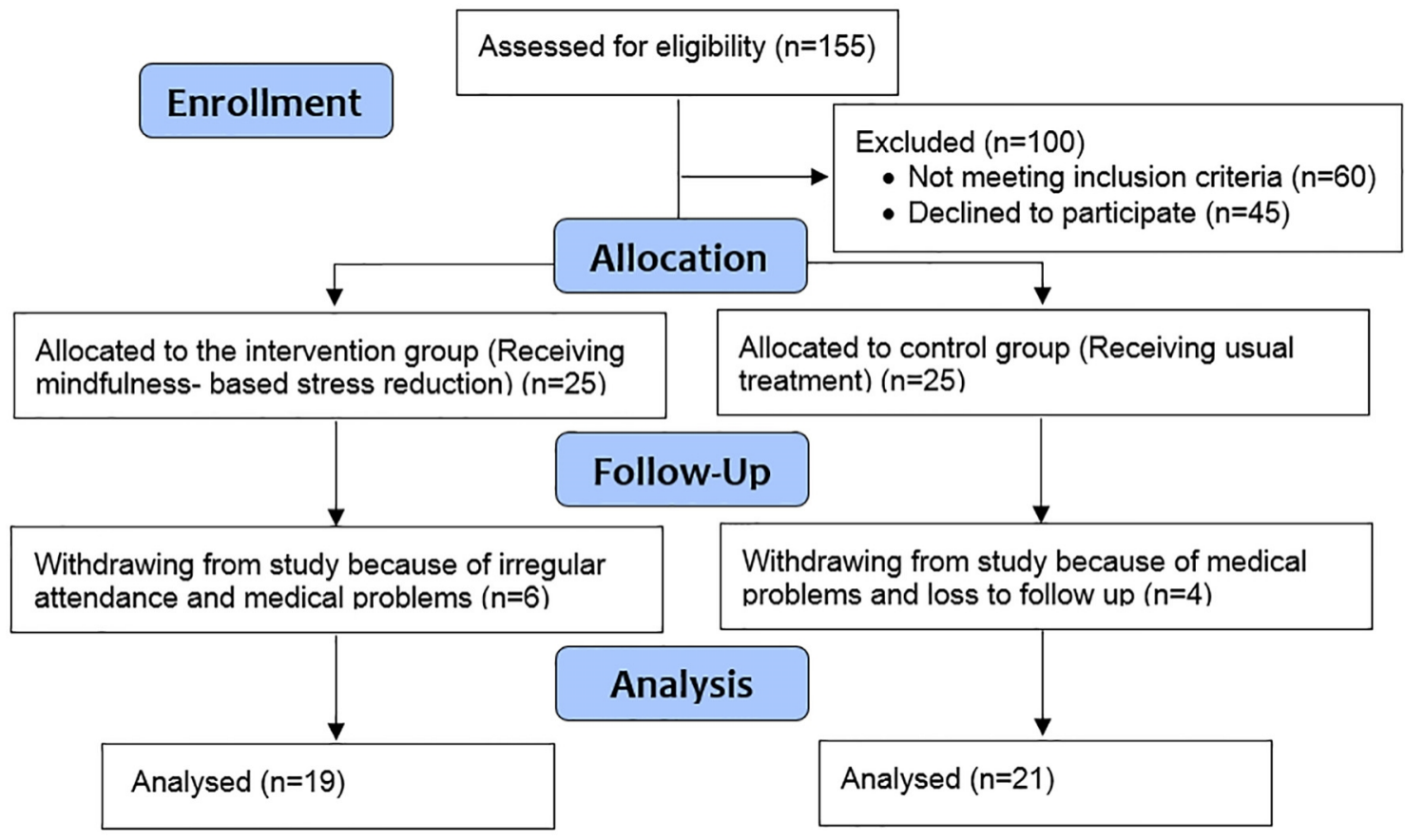

Figure 1. CONSORT 2010 flowchart of the trial 
Table 1. The frequency of demographic characteristics of study groups.

\begin{tabular}{|c|c|c|c|c|c|}
\hline \multirow{2}{*}{\multicolumn{2}{|c|}{ Variables }} & \multicolumn{2}{|l|}{ Groups } & \multirow[t]{2}{*}{ Statistic } & \multirow[t]{2}{*}{ p-value } \\
\hline & & Intervention & Control & & \\
\hline \multicolumn{2}{|c|}{ Age (mean \pm SD) } & $46.8(11.6)$ & $46.2(11.7)$ & 0.15 & 0.87 \\
\hline \multicolumn{2}{|c|}{ Depression (mean \pm SD) } & $7.6(4.0)$ & $7.7(5.3)$ & 0.05 & 0.95 \\
\hline \multicolumn{2}{|c|}{ Anxiety (mean \pm SD) } & $6.7(3.8)$ & $5.6(3.6)$ & -0.99 & 0.32 \\
\hline \multicolumn{2}{|c|}{ Disease duration (mean \pm SD) } & $2.4(1.8)$ & $2.6(1.3)$ & 0.39 & 0.76 \\
\hline \multirow[t]{2}{*}{ Sex } & Male; n (\%) & $12(44)$ & $15(56)$ & \multirow[t]{2}{*}{0.31} & \multirow[t]{2}{*}{0.57} \\
\hline & Female; n (\%) & $7(54)$ & $6(46)$ & & \\
\hline \multirow[t]{3}{*}{ Education } & Elementary \& Guidance school; n (\%) & $10(50)$ & $10(50)$ & \multirow[t]{3}{*}{0.12} & \multirow[t]{3}{*}{0.94} \\
\hline & High school; n (\%) & $6(46)$ & $7(54)$ & & \\
\hline & High educated; $\mathrm{n}(\%)$ & $3(43)$ & $4(57)$ & & \\
\hline \multirow[t]{3}{*}{ Marital status } & Single; n (\%) & $3(50)$ & $3(50)$ & \multirow[t]{3}{*}{0.52} & \multirow[t]{3}{*}{0.86} \\
\hline & Married (\%) & $14(45)$ & $17(55)$ & & \\
\hline & Divorced \& Widowed; n (\%) & $2(66)$ & $1(44)$ & & \\
\hline \multirow[t]{3}{*}{ Job } & Unemployed; n (\%) & $6(46)$ & $7(54)$ & \multirow[t]{3}{*}{0.90} & \multirow[t]{3}{*}{0.63} \\
\hline & Employed; n (\%) & $6(60)$ & $4(40)$ & & \\
\hline & Disabled; n (\%) & $7(41)$ & $10(59)$ & & \\
\hline
\end{tabular}

Table 2. ANCOVA analysis of depression score by MBSR and control groups.

\begin{tabular}{|l|l|l|l|l|l|l|}
\hline Source & $\begin{array}{l}\text { Type III Sum } \\
\text { of Squares }\end{array}$ & df & $\begin{array}{l}\text { Mean } \\
\text { Square }\end{array}$ & F & Sig. & $\begin{array}{l}\text { Partial Eta } \\
\text { Squared }\end{array}$ \\
\hline Corrected Model & $578.3^{\text {a }}$ & 2 & 289.1 & 171.2 & 0.000 & 0.910 \\
\hline Intercept & 1.7 & 1 & 1.7 & 1.0 & 0.311 & 0.030 \\
\hline Baseline depression score & 558.1 & 1 & 558.1 & 330.5 & 0.000 & 0.907 \\
\hline group & 30.4 & 1 & 30.4 & 18.0 & 0.000 & 0.346 \\
\hline Error & 57.4 & 34 & 1.6 & & & \\
\hline Total & 2298.0 & 37 & & & & \\
\hline Corrected Total & 635.7 & 36 & & & & \\
\hline
\end{tabular}

a: R Squared $=0.910$ (Adjusted R Squared $=0.904)$

Table 3. ANCOVA analysis of anxiety score by MBSR and control groups.

\begin{tabular}{|l|l|l|l|l|l|l|}
\hline Source & $\begin{array}{l}\text { Type III Sum } \\
\text { of Squares }\end{array}$ & df & $\begin{array}{l}\text { Mean } \\
\text { Square }\end{array}$ & F & Sig. & $\begin{array}{l}\text { Partial Eta } \\
\text { Squared }\end{array}$ \\
\hline Corrected Model & $321.1 \mathrm{a}$ & 2 & 160.5 & 180.3 & 0.000 & 0.912 \\
\hline Intercept & 1.1 & 1 & 1.1 & 1.2 & 0.272 & 0.034 \\
\hline Baseline Anxiety score & 315.4 & 1 & 315.4 & 354.3 & 0.000 & 0.910 \\
\hline group & 21.7 & 1 & 21.7 & 24.4 & 0.000 & 0.411 \\
\hline Error & 31.1 & 35 & .8 & & & \\
\hline Total & 1353.0 & 38 & & & & \\
\hline Corrected Total & 352.3 & 37 & & & & \\
\hline
\end{tabular}

a. R Squared $=0.912$ (Adjusted R Squared $=0.907)$

\section{Discussion}

In this study, we found a significant reduction in the depression and anxiety scores in hemodialysis patients following the completion of MBSR. Our findings are consistent with the results of other clinical trials in patients with chronic depression and anxiety $(21,22)$, normal population $(23-25)$, and in medical patients with anxiety disorder and recovered recurrently depressed patients $(21,26,27)$. The Reilly-Spong study on the effectiveness of telephone-adapted mindfulness-based stress reduction for patients awaiting kidney transplantation showed reasonable attendance rates and higher expectations in patients following the intervention, and suggested that MBSR would be of use for coping with their health problems in future (28).

A study on 14 patients with chronic disease showed that MBSR reduced blood pressure, depression level, anxiety and psychological distress (29). Williams' study reported significant decreases in the effects of daily hassles, 
psychological distress, and medical symptoms that were maintained at the 3-month follow-up compared to the control group (30). Henderson, in a clinical trial study, examined the effectiveness of the mindfulness-based stress reduction in patients with breast cancer and concluded that depression, anxiety, and coping strategies were significantly different in the intervention group compared to the control group (31). In addition, Carlson et al. found similar results among patients suffering from breast cancer. They proposed that the mindfulness-based stress management program improves depression in patients (32). A study by Omidi showed that MBSR has a positive impact on the quality of life of cardiovascular patients (33). Another study in 2015 by Omidi showed that MBSR in patients with tension headache would reduce their perceived stress and improve their psychological health (34).

The result of our study showed that MBSR has been effective in reducing depression and anxiety symptoms and increasing the quality of life in dialysis patients. Generally, we might expect MBSR to influence psychological outcomes by encouraging the patients to experience the present moment intentionally in a non-judgmental and accepting way, which is the function of mindfulness (35). Additionally, by developing self-observation, the patient can observe his/her thoughts and feelings non-reactively. Therefore, negative reactivity, repetitive negative thinking and over general autobiographical memory retrieval would decrease (36). Results from some studies show that MBSR can alter the function of the brain that is responsible for emotion regulation and the areas that govern how we react to stressful impulses (36-38). Perhaps another reason for the improvement of depression in these patients is the specification of important values and life goals, and concentrating on them instead of being occupied with routine problems and symptoms of chronic kidney disease (39).

\section{Limitations}

There are a number of limitations in this study. The largest limitations include the small sample of 40 subjects. The small sample size limited the amount of power necessary to detect statistical significance. The lack of information about other major health conditions that may have impacted study findings could be seen as another limitation. Future studies would benefit from gaining access to medical history to account for such underlying differences.

\section{Conclusions}

Overall, the present study showed that MBSR can reduce the level of depression and anxiety in hemodialysis patients. This emphasizes the necessity for psychological interventions along with pharmacologic therapy. Hence, to help patients get the most benefit from treatment, MBSR can be used along with pharmacotherapy, to decrease the anxiety and depression in hemodialysis patients.

\section{Acknowledgments:}

The authors would like to thank the Clinical Research Development Unit-Matini/Kargarnejad Hospitals and all the valued patients that helped them in this project. The authors would also like to thank the Research Department of Kashan University of Medical Sciences for financial support of the study (Ref: 9436).

Funding: This study was financially supported by the Deputy of Research, Kashan University of Medical Sciences, Kashan, Iran (Ref: 9436).

Clinical Trial Registration: The protocol of study was registered at the Iranian Registry of Clinical Trials (IRCT) with ID number of IRCT2015081723652N1.

\section{Conflict of Interest:}

There is no conflict of interest to be declared.

Authors' contributions:

All authors contributed to this project and article equally. All authors read and approved the final manuscript.

\section{References:}

1) McCullough K, Sharma P, Ali T, Smith WC, MacLeod A, Black C. Measuring the population burden of chronic kidney disease: a systematic literature review of the estimated prevalence of impaired kidney function. Nephrol Dial Transplant. 2011; 27(5): 1812-21. doi: 10.1093/ndt/gfr547. PMID: 21965592.

2) Mousavi SS, Soleimani A, Mousavi MB. Epidemiology of end-stage renal disease in Iran: a review article. Saudi J Kidney Dis Transpl. .2014; 25(3): 697-702. PMID: 24821181. 
3) Mokhtari N, Nasiri M, Mashuuf T, Kazemnejad E. Comparative study of hemodialysis patients' QOL from patients' and nurses' perception. Journal of Guilan University of Medical Sciences. 2003; 12(47): 16-23.

4) Zamanzadeh V, Heidarzadeh M, Oshvandi KH, Lakdizaji S. Relationship between quality of life and social support in hemodialysis patients in Imam Khomeini and Sina educational hospitals of Tabriz University of medical sciences. Medical Journal of Tabriz University of Medical Sciences. 2007; 29(1): 49-54.

5) Levey AS, Coresh J. Chronic kidney disease. Lancet. 2012; 379: 165-80.

6) Pakpour A, Panahi CH, Yekaninejad M, Ebadi Fard Azar F, Izy H. A comparative study on health related quality of life in hemodialysis patients and healthy people. Journal of Health Administration. 2006; 9(25): $55-62$.

7) Navidian A, Arbabi Sarjou A, Kikhai A. Frequency of mental disturbances in hemodialysis patients referred to hemodialysis ward of khatam-al-anbia hospital in zahedan. Journal of Guilan University of Medical Sciences. 2006; 15(58): 61-7.

8) Nazemian F, GHafari F, Pourghazneyn T. Evaluation of depression and anxiety in hemodialysis patients. Medical journal of mashhad university of medical sciences. 2008; 51(3): 171-6.

9) Hedayati SS, Grambow SC, Szczech LA, Stechuchak KM, Allen AS, Bosworth HB. Physician-diagnosed depression as a correlate of hospitalizations in patients receiving long-term hemodialysis. Am J Kidney Dis. 2005; 46(4): 642-9. doi: 10.1053/j.ajkd.2005.07.002. PMID: 16183419.

10) Feroze U, Martin D, Reina-Patton A, Kalantar-Zadeh K, Kopple JD. Mental health, depression, and anxiety in patients on maintenance dialysis. Iranian journal of kidney diseases. 2010; 4(3): 173-80.

11) Hosseini SH, Espahbodi F, Mirzadeh Goudarzi SM. Citalopram versus psychological training for depression and anxiety symptoms in hemodialysis patients. Iranian journal of kidney diseases. 2012; 6(6): 446-51.

12) Stern TA, Freudenreich O, Smith FA, Fricchione GL, Rosenbaum JF. Hanbook of general hospital psychiatry. 7th edition. 2018: 306-10.

13) Ie A, Ngnoumen CT, Langer EJ. The Wiley Blackwell handbook of mindfulness. John Wiley \& Son; 2014.

14) Omidi A, Mohammadi A, Zargar F, Akbari H. Efficacy of Mindfulness-Based Stress Reduction on Mood States of Veterans With Post-Traumatic Stress Disorder. Arch Trauma Res. 2013; 1(4): 151-4.

15) Bränström R, Duncan LG, Moskowitz JT .The association between dispositional mindfulness, psychological well - being, and perceived health in a Swedish population - based sample. Br J Health Psychol. 2011; 16(2): 300-16. doi: 10.1348/135910710X501683. PMID: 21489058, PMCID: PMC3762484.

16) Omidi A, ZargarF. Effect of mindfulness-based stress reduction on pain severity and mindful awareness in patients with tension headache: a randomized controlled clinical trial. Nurs Midwifery stud. 2014;3(3): e21136. PMID: 25699282, PMCID: PMC4332994.

17) Kolahkaj B, ZargarF. Effect of mindfulness-based stress reduction on anxiety, depression and stress in women with multiple sclerosis. Nursing and midwifery studies. 2015; 4(4): e29655. doi: 10.17795/nmsjournal29655. PMID: 26835467, PMCID: PMC4733502.

18) Merkes M. Mindfulness-based stress reduction for people with chronic diseases. Aust J Prim Health. 2010; 16(3): 200-10. doi: 10.1071/PY09063. PMID: 20815988.

19) Parswani MJ, Sharma MP, Iyengar S. Mindfulness-based stress reduction program in coronary heart disease: A randomized control trial. Int J Yoga. 2013 Jul;6(2):111-7. doi: 10.4103/0973-6131.113405. PMID: 23930029, PMCID: PMC3734636

20) Montazeri A, Vahdaninia M, Ebrahimi M. The Hospital Anxiety and Depression Scale (HADS): translation and validation study of the Iranian version. Health Qual Life Outcomes. 2003; 1(1): 14. doi: 10.1186/14777525-1-14. PMID: 12816545, PMCID: PMC161819.

21) Peterson LG, Pbert L. Effectiveness of a meditation-based stress reduction program in the treatment of anxiety disorders. Am J Psychiatry. 1992; 149(7): 936-43.

22) Barnhofer T, Crane C, Hargus E, Amarasinghe M, Winder R, Williams JM. Mindfulness- based cognitive therapy as a treatment for chronic depression: A preliminary study. Behav Res Ther. 2009; 47(5): 366-73. doi: 10.1016/j.brat.2009.01.019. PMID: 19249017, PMCID: PMC2866254.

23) Bohlmeijer E, Prenger R, Taal E, Cuijpers P. The effects of mindfulness-based stress reduction therapy on mental health of adults with a chronic medical disease: a meta-analysis. J Psychosom Res. 2010; 68(6): 539-44. doi: 10.1016/j.jpsychores.2009.10.005. PMID: 20488270.

24) Speca M, Carlson LE, Goodey E, Angen M. A randomized, wait-list controlled clinical trial: the effect of a mindfulness meditation-based stress reduction program on mood and symptoms of stress in cancer. Psychosom Med. 2000; 62(5): 613-22. PMID: 11020090. 
25) Reibel DK, Greeson JM, Brainard GC, Rosenzweig S. Mindfulness-based stress reduction and healthrelated quality of life in a heterogeneous patient population. Gen Hosp Psychiatry. 2001; 23(4): 183-192. doi: 10.1016/S0163-8343(01)00149-9. PMID: 11543844.

26) Miller JJ, Fletcher K, Kabat-Zinn J. Three-year follow-up and clinical implications of a mindfulness meditation-based stress reduction intervention in the treatment of anxiety disorders. General hospital psychiatry. 1995; 17(3): 192-200. PMID: 7649463

27) Teasdale JD, Segal ZV, Williams JM, Ridgeway VA, Soulsby JM, Lau MA. Prevention of relapse/recurrence in major depression by mindfulness-based cognitive therapy. J Consult Clin Psychol. 2000; 68(4): 615-23. PMID: 10965637.

28) Reilly-Spong M, Reibel D, Pearson T, Koppa P, Gross CR. Telephone-adapted mindfulness-based stress reduction (tMBSR) for patients awaiting kidney transplantation: Trial design, rationale and feasibility. Contemporary clinical trials. 2015; 42: 169-184. doi: 10.1016/j.cct.2015.03.013. PMID: 25847578, PMCID: PMC4450136.

29) Astin JA. Stress reduction through mindfulness meditation. Psychotherapy and psychosomatics. 1997; 66(2): 97-106. doi: 10.1159/000289116.

30) Williams KA, Kolar MM, Reger BE, Pearson JC. Evaluation of a wellness-based mindfulness stress reduction intervention: A controlled trial. AJHP. 2001; 15(6): 422-432. doi: 10.4278/0890-1171-15.6.422.

31) Henderson VP, Clemow L, Massion AO, Hurley TG, Druker S, Hébert JR. The effects of mindfulnessbased stress reduction on psychosocial outcomes and quality of life in early-stage breast cancer patients: a randomized trial. Breast Cancer Res Treat. 2012; 131(1): 99-109. doi: 10.1007/s10549-011-1738-1. PMID: 21901389 , PMCID: PMC3784652.

32) Carlson LE, Doll R, Stephen J, Faris P, Tamagawa R, Drysdale E, et al. Randomized-controlled multi-site trial of mindfulness-based cancer recovery (MBCR) versus supportive expressive group therapy (SET) among breast cancer survivors (MINDSET): long-term follow-up results. Pscyho-oncology. 2013; 22: 103104. doi: 10.1200/JCO.2012.47.5210.

33) Omidi A, Momeni J, Raygan F, Akbari H, Talighi E. The effectivness of mindfulness-based stress reduction program on quality of life among patients with cardiovascular disease. Journal of Thought and behavioral in clinical psychology. 2017; 10(24): 7-16.

34) Omidi A, Zargar F. Effect of mindfulness-based stress reduction on percived stress and psychological health in patients with tension headache. JRMS. 2015; 20: 1058-63.

35) Kabat-Zinn J. Wherever you go, there you are: Mindfulness meditation in everyday life. New York: Hyperion.

36) Gu J, Strauss C, Bond R, Cavanagh K. How do mindfulness-based cognitive therapy and mindfulnessbased stress reduction improve mental health and wellbeing? A systematic review and meta-analysis of mediation studies. Clinical Psychology Review. 1994; 37(2015): 1-12.

37) Daividson RJ. Kabat-Zinn J, Schumacher J, Rosenkranz M, Muller D, Santorelli SF, et al. Alteration in brain and immune function produced by mindfulness meditation. Psychosom Med. 2003; 65: 564-40. PMID: 12883106.

38) Lazar SW, Kerr CE, Wasserman RH, Gray JR, Greve DN, Treadway MT, et al. Meditation experience is associated with increased cortical thickness. Neuroreport. 2005; 16: 1893-7. PMID: 16272874, PMCID: PMC1361002.

39) Kimmel PL. Psychosocial factors in adult end-stage renal disease patients treated with hemodialysis: correlates and outcomes. Am J Kidney Dis. 2000; 35(4): S132-40. doi: 10.1016/S0272-6386(00)70240-X. PMID: 10766011. 